\title{
TYPE Ia SUPERNOVAE: CAN CORIOLIS FORCE BREAK THE SYMMETRY OF THE GRAVITATIONAL CONFINED DETONATION EXPLOSION MECHANISM?
}

\author{
D. García-Senz ${ }^{1,2}$, R. M. Cabezón ${ }^{3}$, I. Domínguez ${ }^{4}$, and F. K. Thielemann ${ }^{3}$ \\ ${ }^{1}$ Departament de Física, UPC, Comte d'Urgell 187, E-08036 Barcelona, Spain; domingo.garcia@upc.edu \\ ${ }^{2}$ Institut d'Estudis Espacials de Catalunya, Gran Capità 2-4, E-08034 Barcelona, Spain \\ ${ }^{3}$ Departement Physik, Universität Basel. Klingelbergstrasse, 82, 4056 Basel, Switzerland; ruben.cabezon@unibas.ch \\ ${ }^{4}$ Departamento de Física, Teórica y del Cosmos, Universidad de Granada, E-18071 Granada, Spain \\ Received 2015 July 17; accepted 2016 January 30; published 2016 March 8
}

\begin{abstract}
Currently the number of models aimed at explaining the phenomena of type Ia supernovae is high and distinguishing between them is a must. In this work we explore the influence of rotation on the evolution of the nuclear flame that drives the explosion in the so-called gravitational confined detonation models. Assuming that the flame starts in a pointlike region slightly above the center of the white dwarf (WD) and adding a moderate amount of angular velocity to the star we follow the evolution of the deflagration using a smoothed particle hydrodynamics code. We find that the results are very dependent on the angle between the rotational axis and the line connecting the initial bubble of burned material with the center of the WD at the moment of ignition. The impact of rotation is larger for angles close to $90^{\circ}$ because the Coriolis force on a floating element of fluid is maximum and its principal effect is to break the symmetry of the deflagration. Such symmetry breaking weakens the convergence of the nuclear flame at the antipodes of the initial ignition volume, changing the environmental conditions around the convergence region with respect to non-rotating models. These changes seem to disfavor the emergence of a detonation in the compressed volume at the antipodes and may compromise the viability of the so-called gravitational confined detonation mechanism.
\end{abstract}

Key words: hydrodynamics - methods: numerical - supernovae: general

\section{INTRODUCTION}

Because of the many connections of type Ia supernovae (SNe Ia) to fundamental problems in astrophysics such as, for example, the chemical evolution of galaxies (Iwamoto et al. 1999; Seitenzahl et al. 2013) or the origin of the acceleration of the universe (Riess et al. 1998), the quest for the mechanism behind these explosions is of utmost relevance. There is a general agreement that an SN display involves the explosion of a white dwarf (WD), but beyond this point there is not an agreement on the details. The explosion may be the outcome of the destabilization of a WD that approaches the Chandrasekhar mass limit owing to mass accretion from a nearby companion star, a succession of events referred as the single degenerate scenario (SD; Whelan \& Iben 1973; Nomoto 1982). Another route to the explosion involves the merging of two WDs with more or less canonical masses, $M_{\mathrm{WD}} \simeq 0.7 M_{\odot}$, orbiting in a close binary system (Iben \& Tutukov 1984; LorénAguilar et al. 2009; Pakmor et al. 2013), which is known as the double degenerate scenario (DD). This scenario includes the direct collision of two WDs as a limiting case (Rosswog et al. 2009; García-Senz et al. 2013). Actually, both channels may coexist and contribute to the observed averaged rate of SNe Ia (Wang \& Han 2012). However, each scenario, SD or DD, exhibits a considerable degree of degeneracy because the basic observational properties of $\mathrm{SNe}$ Ia are matched by different explosion models. Excluding violent mergers and He detonations, there is not a consensus about the mechanism driving these explosions (Branch \& Khokhlov 1995; Hillebrandt \& Niemeyer 2000; Hillebrandt et al. 2013). Among the different mechanisms found in the literature there are either pure (subsonic) deflagrations (Nomoto et al. 1984; Fink et al. 2014) or deflagrations which, at some critical density, turn into a (supersonic) detonation (Khokhlov 1991a), although the physical agent driving the deflagration to detonation transition is uncertain. Another way to detonate the WD relies on the gravitational confinement of the plasma (the cosmic version of the terrestrial inertial confinement fusion). In these confinement scenarios there is a brief deflagrative phase that causes the expansion of the WD before a large amount of material is burned. The explosion is rather weak which results in a failed $\mathrm{SNe}$ Ia. Still, the explosion can be revitalized in two ways leading to the pulsating and gravitational confined models of thermonuclear SNe. The pulsating explosion models arise after the fallback of some material previously expelled and the formation of an accretion shock that compresses and heats the core. In steady conditions the ram pressure exerted by the infalling material keeps the plasma compressed long enough to cause temperature conditions for which ${ }^{12} \mathrm{C}+{ }^{12} \mathrm{C}$ is active, which, under the appropriate physical conditions, may give rise to a Chapman-Jouguet detonation which incinerates the whole core. Because in these scenarios the explosion ensues after a global pulsation of the WD, they were called pulsating delayed detonation models in spherically symmetric calculations (Ivanova et al. 1974; Khokhlov 1991b) and pulsating reverse detonations (PRD) in three dimensions (3D; Bravo \& GarcíaSenz 2006, 2009; Bravo et al. 2009). A second possibility is that considered in the gravitational confined detonation models (GCD; Plewa et al. 2004; Plewa 2007; Röpke et al. 2007; Townsley et al. 2007; Jordan et al. 2008; Meakin et al. 2009; Seitenzahl et al. 2009a) whose main features are: (1) the deflagration flame ignites in a small volume offset from the center of the WD, (2) the bubble of hot fluid accelerates vertically by buoyancy in the strong gravitational field of the compact object, (3) soon the bubble made of ashes expands over the surface of the star and converges at the opposite side from where the initial breakout occurred, and (4) as the 
convergence at the antipodes is strong, it produces an inwardly moving jet that may give rise to detonation in (or close to) the core.

Until now all GCD models have been calculated assuming that the WD is not rotating at the moment of the explosion. Nevertheless, we know that in both the SD and the DD scenarios, the WD could store an important amount of rotation because of the angular momentum transferred from the accretion disk to the WD (Piersanti et al. 2003; Yoon \& Langer 2004, 2005). In GCD models the deflagrative phase sets the conditions of the detonation at late times, and it is very likely that the rotation axis is not perfectly aligned with the symmetry plane of the flame. As a consequence, Coriolis force will act with different strengths in different regions of the burning material. Therefore, to what extent may rotation weaken the efficiency of the convergence of the ashes at the antipodes?

Although common proposed scenarios of SNe Ia imply the rotation of a WD, the number of multidimensional calculations of the explosion that incorporate rotation is really scarce. One of them was carried out by Steinmetz et al. (1992) simulating the detonation of a fast rotating WD. More recently, Pfannes et al. (2010b) calculated the deflagration of a rapidly spinning WD in 3D after the central ignition of the fuel and concluded that the yields of the species synthesized during the explosion do not match the observed spectra. In a subsequent work, the same authors (Pfannes et al. 2010a) analyzed the detonation of a fast rotating WD and concluded that this mechanism could explain the existence of some superluminous $\mathrm{SNe}$ Ia.

In the present work we analyze for the first time the possible consequences of including the rotation in the GCD scenario. To do that we have carried out several 3D simulations using a smoothed particle hydrodynamics (SPH) code with moderate resolution. The amount of rotation considered in our exploratory study is modest, $\Omega / \Omega_{c} \simeq 0.08$, where $\Omega_{c} \simeq 4.7 \mathrm{~s}^{-1}$ is the critical Keplerian velocity at the equator, so as to not appreciably modify the spherically symmetric density profile of the WD at the moment of the explosion. As we will see, even that small amount of rotation is able to change the thermodynamic conditions at the convergence point of the ashes. Therefore, future studies dealing with confinement scenarios should incorporate rotation as a basic ingredient, adding a step of difficulty to an already complex problem.

In Section 2 we describe the main features of the hydrocode, the initial setting, and the method to calculate the evolution of the nuclear flame (described with more detail in the Appendix). We also provide here a brief discussion of the profile of the angular velocity adopted in the simulations. A detailed description of the hydrodynamic evolution of the four considered models is given in Section 3. Finally, Section 4 summarizes the main conclusions of our work.

\section{HYDRODYNAMIC METHOD. FLAME HANDLING AND INITIAL SETTING}

In the GCD and PRD scenarios, the final explosion of the WD takes place after a considerable change in the WD radius. SPH is a Lagrangian method free of numerical diffusion and is well suited to handle systems hosting a variety of dynamical scales (Rosswog 2009; Springel 2010) and to track the complex geometry of the nuclear flame which powers the explosion. As angular momentum is exactly conserved in SPH, this technique is also adequate to handle problems involving rotation. We are using an updated version of the SPH code devised more than a decade ago which was applied to simulate the thermonuclear explosion of a WD by different physical mechanisms, either deflagrations (García-Senz \& Bravo 2005) or detonations (Bravo \& García-Senz 2008). Nevertheless, our new hydrocode SPHYNX (R. M. Cabezón et al., in preparation), incorporates a large number of state-of-the-art improvements that are worth mentioning. The most relevant update concerns gradient estimation, which now relies on an integral approach that is more accurate than the traditional method in SPH (García-Senz et al. 2012; Cabezón et al. 2012). To carry out interpolations, SPHYNX makes use of the sinc family of kernels (Cabezón et al. 2008), which are more resistant to particle clustering than the standard cubic spline, therefore allowing one to increase the number of interpolating particles in the SPH summations to reduce the numerical noise. The smoothing length $h(\boldsymbol{r}, t)$ is updated according to the method by Springel \& Hernquist (2002), which ensures both energy and entropy conservation. In addition the code has been parallelized with a hybrid scheme in MPI + openMP, so that we are routinely using $\simeq 2 \times 10^{6}$ particles.

The algorithm to track the flame still relies on a diffusionreaction scheme but it incorporates some novel improvements. Among them, a phenomenological subgrid model to estimate the characteristic turnover velocity, $v_{t}$, of the eddies at scales resolved by the hydrocode (see the Appendix). The effective velocity of the flame is $v_{f}=\max \left(v_{l}, v_{t}\right)$, with $v_{l}$ being the laminar velocity of the flame from Timmes \& Woosley (1992). At the typical conditions considered in our simulations we obtain $100 \mathrm{~km} \mathrm{~s}^{-1} \lesssim v_{f} \lesssim 500 \mathrm{~km} \mathrm{~s}^{-1}$, the former being roughly the conductive flame velocity at $\rho \simeq 2 \times 10^{9} \mathrm{~g} \mathrm{~cm}^{-3}$, and the latter only achieved in points where the shear is high. Different groups approximate $v_{f}$ using a variety of numerical schemes, based either on a careful estimation of the turbulent velocity plus the level-set approach (Reinecke et al. 1999) or in diffusion-reaction schemes either with constant propagation velocity (García-Senz \& Bravo 2005; Ma et al. 2013) or powered by the Rayleigh-Taylor instability (Gamezo et al. 1999; Plewa et al. 2004; Plewa 2007). It is difficult to ascertain to what extent the gross details of the evolution depend on the precise value of $v_{f}$. The effective flame velocity may selfregulate through the creation/destruction of the heat-exchanging surface between ashes and fuel, as seen in the numerical experiments in boxes by Khokhlov (1995). Nevertheless, that mechanism is doubtful in an unconfined media, especially in those cases where much of the combustion takes place after a considerable expansion of the star (as in the GCD model). A glance at Figure 3 shows that in the GCD models a large amount of nuclear energy is released around $t \simeq 0.9-1 \mathrm{~s}$ when the surface of the flame is large. At this point, the combustion also competes with the expansion of the WD, which soon causes the freeze-out of the nuclear reactions. A faster effective flame velocity releases more nuclear energy and provokes the prompt expansion of the star, quenching the combustion. In this sense, the trade-off between these two antagonistic effects may qualitatively act as a regulating valve of the explosion.

The physics included is very similar to that recently used by García-Senz et al. (2013) to simulate the collision of WDs. The nuclear network is an $\alpha$-chain complemented with carbon and oxygen binary reactions. The evolution of the species is calculated implicitly with the temperature to ensure a smooth transition to the nuclear statistical equilibrium (NSE) regime 
(Cabezón et al. 2004) (see more details in the Appendix). Electron captures on protons and nuclei have been neglected because their impact on the dynamics of the explosion is secondary (Meakin et al. 2009). Our EOS has the contributions of electrons (Blinnikov et al. 1996), ions (including Coulomb and polarization corrections), and radiation.

The initial model is a WD with central density $\rho_{c}=2.6 \times 10^{9} \mathrm{~g} \mathrm{~cm}^{-3}$, homogeneous temperature $T=2 \times$ $10^{7} \mathrm{~K}$, and composition $X_{C}=X_{O}=0.5$. The integration of the Lane-Emden equation for these conditions gives a mass of $M_{\mathrm{WD}}=1.376 M_{\odot}$. The WD was then mapped to a 3D configuration of particles and relaxed to get rid of the excess of numerical noise before starting the hydrodynamic simulations. We proceeded in two steps to relax the initial models. First, the sample of particles is spread in the radial direction, according to the one-dimensional density profile and randomly distributed in the spherical angles $\theta, \varphi$. We then let the particles move, suppressing the radial component of the velocity so that they displace tangentially until the density profile approaches the analytical one. In this way, we erase spurious clumps of particles originating from the random distribution. At this point, the much longer second stage starts. The sample of particles is allowed to move in any direction so that they try to find the configuration of minimum energy. To remove the excess of energy stored as numerical noise we add a damping force proportional to the velocity. As a result, the WD settles in a stable structure with the correct central density usually after several seconds of evolution. Taking $N=2 \times 10^{6}$ the maximum resolution is $\simeq 12 \mathrm{~km}$ achieved at the center of the WD. While this resolution is high enough to capture the main features of the explosion during the deflagration phase, it is below what is needed to study the transition to a possible detonation at late times.

All calculations reported in this paper assume that the thermonuclear ignition of the WD starts in a single small region (a bubble) close to the center of the star and propagates to the remaining plasma by hydrodynamic instabilities. This initial setting is similar to that currently used by other groups, easing the comparison of results (Röpke et al. 2007; Meakin et al. 2009). On another note, the ignition in a single region at an altitude $\simeq 50 \mathrm{~km}$ was favored in recent studies of the preignition stage by Zingale et al. (2011), Nonaka et al. (2012), and Malone et al. (2014) using the MAESTRO code. In several of these works, slow rotation was shown to affect the long convective phase preceding the ignition (Zingale et al. 2011; see also Kuhlen et al. 2006). We also assume that before the convergence of the ashes the combustion regime is purely deflagrative; that is, we have ignored any potential deflagration to detonation transition when the flame enters into the distributed regime at densities $\rho \simeq 2 \times 10^{7} \mathrm{~g} \mathrm{~cm}^{-3}$ (Khokhlov 1991a; Gamezo et al. 2005).

The main goal of this work is to explore the impact of rotation on the GCD models. Nevertheless, we have restricted the study to moderate rotators to preserve the spherical symmetry and equilibrium properties of the initial model, which is the same for all calculated models. Considering faster rotators would require a totally different initial setting, which is beyond the scope of the present manuscript. The main features of the calculated models during the deflagration phase are given in Table 1.
The rotational velocity of a WD at the end of the accretion phase is poorly known. There is some agreement that the core is probably rotating as a rigid body and, beyond that, the angular velocity may increase up to a critical radius $R_{c}$, to finally decay to Keplerian values at the surface of the WD (Yoon \& Langer 2004; Pfannes et al. 2010b). Nevertheless, in those cases where an efficient redistribution of angular momentum is assumed, the WD may approach rigid rotation (Piersanti et al. 2003). Rigid rotators could be the result of having either strong braking magnetic torques or efficient viscous angular momentum transport and/or long timescales (Piro 2008).

In our exploratory study we have considered four kinds of models (Table 1): no rotation, rigid rotators with $\Omega_{z}=0.4 \mathrm{~s}^{-1}$ and $\Omega_{x}=0.4 \mathrm{~s}^{-1}$, respectively, and $\Omega_{x}$,

$$
\Omega_{x}=\left\{\begin{array}{cl}
\Omega_{0}=0.6 & r \leqslant R_{c} \\
\frac{\Omega_{0}}{R_{c}-R_{\mathrm{WD}}}\left(r-R_{\mathrm{WD}}\right) & r>R_{c}
\end{array}\right.
$$

where $r$ is the distance to the center and $R_{c}=5 \times 10^{7} \mathrm{~cm}$. This last case is a simple profile consisting of a rigid body rotation near the center which decays linearly to a much lower value at the surface. The case $\Omega_{z} \neq 0$ sets the rotating axis aligned to the initial ascending direction of the bubble, whereas $\Omega_{x} \neq 0$ takes that axis orthogonal to the initial displacement of the bubble. It is in the latter case where we expect the maximum effect of the rotation, mainly driven by the Coriolis force. The value of this acceleration, $\boldsymbol{a}_{\mathrm{Cor}}=2 \boldsymbol{v} \times \Omega$ acting on a piece of a floating bubble, is one of the greatest Coriolis forces found in nature. To have an estimate of the order of magnitude, we can take $\Omega=0.4 \mathrm{~s}^{-1}$ (models $\mathrm{B}, \mathrm{C}, \mathrm{F}$, and I of Table 1) and a typical rising velocity of a bubble $v \simeq 2.5 \times 10^{8} \mathrm{~cm} \mathrm{~s}^{-1}$. This yields $\boldsymbol{a}_{\mathrm{Cor}} \simeq 2 \times 10^{8} \mathrm{~cm} \mathrm{~s}^{-2}$. A huge value, almost comparable to the effective acceleration of the bubble imparted by the Archimedean force $a_{\mathrm{eff}}=g \frac{\Delta \rho}{\rho}$.

In Figure 1 we show several magnitudes related to rotation. As we can see, neither the rotational linear velocity nor the centripetal force are large enough to significantly break the spherical symmetry of the initial model. The largest ratio between the linear velocity and the local escape velocity is $\simeq 5 \%$, obtained just on the equator at the surface of the WD. The ratio between the centripetal force and the floating force acting on a rising blob $g_{\text {eff }}=(\Delta \rho / \rho) g(r) \simeq 0.2 g(r)$, always remains below $3 \%$. Thus, the perturbation introduced by a moderate amount of rotation hardly affects the stability of the initial model during the first tenths of seconds of flame propagation. At longer times the nuclear energy released during the explosion is more powerful than the kinetic rotational energy by several orders of magnitude. The ratio between the rotational kinetic energy and the binding energy of the WD at $t=0$ is also small, $E_{\mathrm{rot}} / E_{\mathrm{bin}} \leqslant 0.2 \%$ in all models. However, the ratio between the Coriolis and floating forces can be as high as $10 \%$ during the first tenths of seconds of flame propagation and should not be disregarded. Moreover, the Coriolis force is constantly pushing the bubble during its race to the antipodes and the cumulative effect may break the symmetry of the convergence of the ashes, especially when the rotational axis is not aligned with the initial line of displacement of the bubble. 
Table 1

Main Features of Both the Initial Models and the Deflagration Phase

\begin{tabular}{|c|c|c|c|c|c|c|c|c|c|c|}
\hline Model & $\begin{array}{c}N \\
10^{6} \text { part } \\
\end{array}$ & $\begin{array}{r}z_{0} \\
\mathrm{~km}\end{array}$ & $\begin{array}{c}r_{b}^{0} \\
\mathrm{~km}\end{array}$ & $\begin{array}{r}\left(\Omega_{x}, \Omega_{y}, \Omega_{z}\right) \\
s^{-1}\end{array}$ & $\begin{array}{c}E_{\text {nuc }} \\
10^{51} \mathrm{erg} \\
\end{array}$ & $\begin{array}{l}M_{\mathrm{Ni}} \\
M_{\odot} \\
\end{array}$ & $\begin{array}{c}\rho_{c}^{\min } \\
10^{7} \mathrm{~g} \mathrm{~cm}^{-3} \\
\end{array}$ & $\begin{array}{r}h_{c} \\
\mathrm{~km}\end{array}$ & $\begin{array}{c}\rho_{T_{9=3}}^{\mathrm{jet}} \\
10^{7} \mathrm{~g} \mathrm{~cm}^{-3} \\
\end{array}$ & $\begin{array}{c}t_{T_{9}=3} \\
\mathrm{~s} \\
\end{array}$ \\
\hline A & 2.0 & 60 & 40 & $(0.0,0.0,0.0)$ & 0.155 & 0.090 & 4.6 & 49 & 1.78 & 4.24 \\
\hline B & 2.0 & 60 & 40 & $(0.0,0.0,0.4)$ & 0.157 & 0.091 & 4.5 & 49 & 1.36 & 4.28 \\
\hline C & 2.0 & 60 & 40 & $(0.4,0.0,0.0)$ & 0.155 & 0.089 & 4.7 & 48 & 1.16 & 4.18 \\
\hline D & 2.0 & 60 & 40 & $(\leqslant 0.6,0.0,0.0)$ & 0.156 & 0.092 & 4.4 & 43 & 1.64 & 4.48 \\
\hline $\mathrm{E}$ & 4.0 & 60 & 40 & $(0.0,0.0,0.0)$ & 0.167 & 0.088 & 3.9 & 36 & 1.44 & 4.40 \\
\hline $\mathrm{F}$ & 4.0 & 60 & 40 & $(0.4,0.0,0.0)$ & 0.149 & 0.097 & 4.8 & 33 & 1.27 & 4.21 \\
\hline G & 4.0 & 60 & 40 & $(\leqslant 0.6,0.0,0.0)$ & 0.162 & 0.095 & 4.1 & 35 & 1.22 & 4.35 \\
\hline $\mathrm{H}$ & 8.0 & 60 & 40 & $(0.0,0.0,0.0)$ & 0.145 & 0.085 & 4.7 & 27 & 1.95 & 4.14 \\
\hline I & 8.0 & 60 & 40 & $(0.4,0.0,0.0)$ & 0.144 & 0.084 & 4.9 & 26 & 1.91 & 4.15 \\
\hline
\end{tabular}

Note. The meaning of the columns is as follows: $N$, number of SPH particles; $z_{0}$ ignition altitude; $r_{b}^{0}$ initial radius of the bubble; $\Omega_{x}, \Omega_{y}, \Omega_{z}$ components of the angular velocity at $t=0 \mathrm{~s} ; E_{\text {nuc }}$ released nuclear energy; $M_{\mathrm{Ni}}$ produced yields of nickel; $\rho_{c}^{\min }, h_{c}\left(\rho_{c}^{\mathrm{min}}\right)$ absolute minimum density and resolution achieved at the center of the core of the WD; $\rho_{T_{9}=3}^{\text {jet }}, t_{T_{9}=3}$ density in the colliding region when the temperature of a particle made of fuel exceeds $3 \times 10^{9} \mathrm{~K}$, and the elapsed time to reach that temperature, respectively.

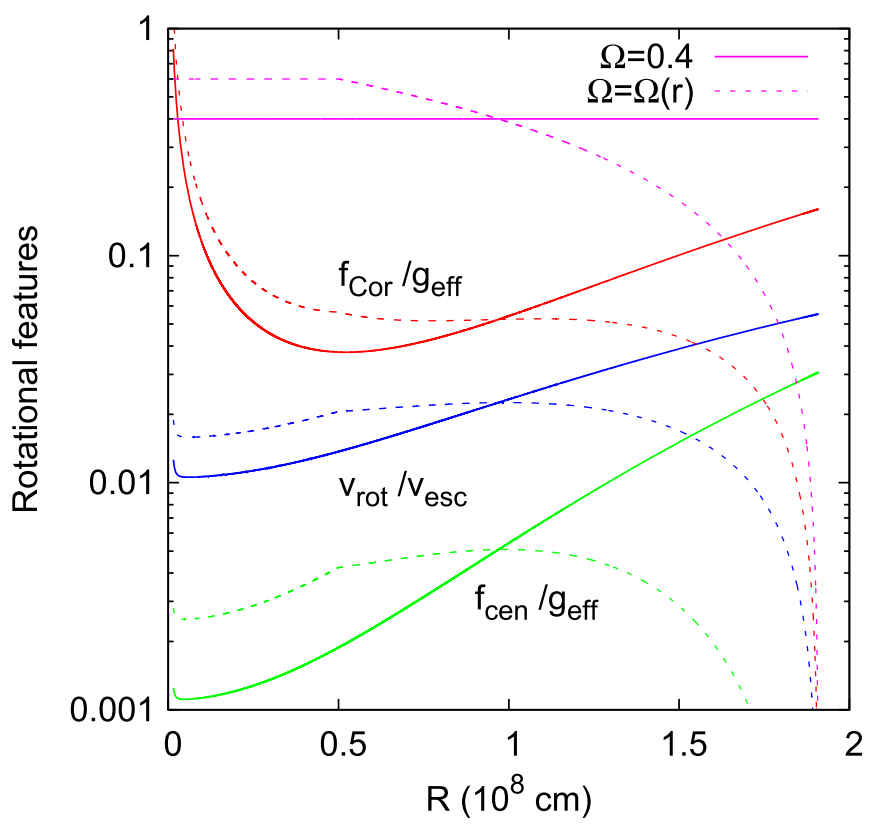

Figure 1. Dimensionless indicators of the equatorial rotational strength for two choices of the angular velocity $\Omega$ of the WD. Continuum lines are for rigid rotators with $\Omega=0.4 \mathrm{~s}^{-1}$. Dashed lines correspond to $\Omega(r)$ calculated with Equation (1).

\section{HYDRODYNAMIC SIMULATIONS}

\subsection{The Deflagration Phase}

Model A in Table 1 was calculated assuming that the WD is not rotating when the first sparks ignite and serves as a reference model. It is also assumed that these seminal sparks ignite in a pointlike region close to (but not at) the center of the core. In all simulated models the geometry of the ignited region is spherical with an initial radius of $20 \mathrm{~km}$. The center of mass of the hot bubble is located $60 \mathrm{~km}$ above the center of the WD. To provoke the ignition the content of the bubble is isochorically burned to NSE, so that the temperature jumps to $\simeq 9$ billion degrees, giving birth to a thermal wave. We let the conductive flame grow during $t=0.25 \mathrm{~s}$ keeping the WD structure frozen to ensure smooth conditions around the flame. At that time the radius of the hot volume has grown up to $40 \mathrm{~km}$. Afterward, the time is reset to $t=0 \mathrm{~s}$ and the hydrodynamic calculation of the explosion starts. Note that even though the incinerated volume at $t=0$ is not perfectly spherical owing to the dependence of the conductive heat transport on density, it still retains the symmetry around the line joining the center of mass of the WD with that of the hot bubble. Nevertheless, the interaction of the residual numerical noise of the WD with the flame gives rise to non-symmetric structures and the perfect axisymmetric geometry of the burned region is lost at later times. Note that some symmetry breaking of the flame is also expected on a physical basis owing to the existence of convective cells moving at several dozens of kilometers per second in the WD at the moment of the explosion. While some amount of numerical noise is needed to power the hydrodynamic instabilities that drive the explosion, its precise impact on the models shown in Table 1 is unpredictable, but it contributes to the $7 \%$ scatter in $E_{\text {nuc }}$ around the central value $\simeq 0.15510^{51} \mathrm{erg}$. Shortly after $t=0 \mathrm{~s}$ the bubble expands, trying to regain equilibrium, and its density drops below that of the surrounding plasma. It begins to float, acquiring a buoyant velocity $v \simeq 2500 \mathrm{~km} \mathrm{~s}^{-1}$ at $t=0.5 \mathrm{~s}$. Soon the interaction of the rising blob with the environment moves its spherical geometry to a toroidal one (see the leftmost panels in Figure 2). From this moment on, the flame accelerates due to both the increase of the effective surface between ashes and fuel and the strong vorticity induced by the Kelvin-Helmholtz instability at the edges of the rising blob. This last effect is handled using the toy model described in the Appendix.

In Figure 3 we show the released nuclear energy, $E_{\text {nuc }}(t)$ and kinetic energy for all four calculated models. The evolution of models A, B, C, and D is very similar, all of them releasing the same amount of nuclear energy. The nuclear energy release shows a strong rise at $t>0.8 \mathrm{~s}$ and begins to saturate at $t \simeq 1.1 \mathrm{~s}$, owing to the expansion of the WD. At the elapsed time $t=1.5 \mathrm{~s}$ all nuclear reactions are virtually quenched while the ashes are surfing around the core of the WD in their journey to the meeting point at the south pole, Figures 2 and 4 . At $t=2.5 \mathrm{~s}$ the nuclear reactions are switched off from the hydrocode to make the analysis of the convergence of the ashes easier. Although the injected energy $E_{\text {nuc }} \simeq 1.5 \times 10^{50} \mathrm{erg}$ is not enough to unbind the star, it provokes a large change in its radius, and the central density drops to $\rho_{c}=4.6 \times 10^{7} \mathrm{~g} \mathrm{~cm}^{-3}$ at $t=3.02 \mathrm{~s}$ (model A). The value of $E_{\text {nuc }}$ is in the range of values obtained in other $3 \mathrm{D}$ calculations 

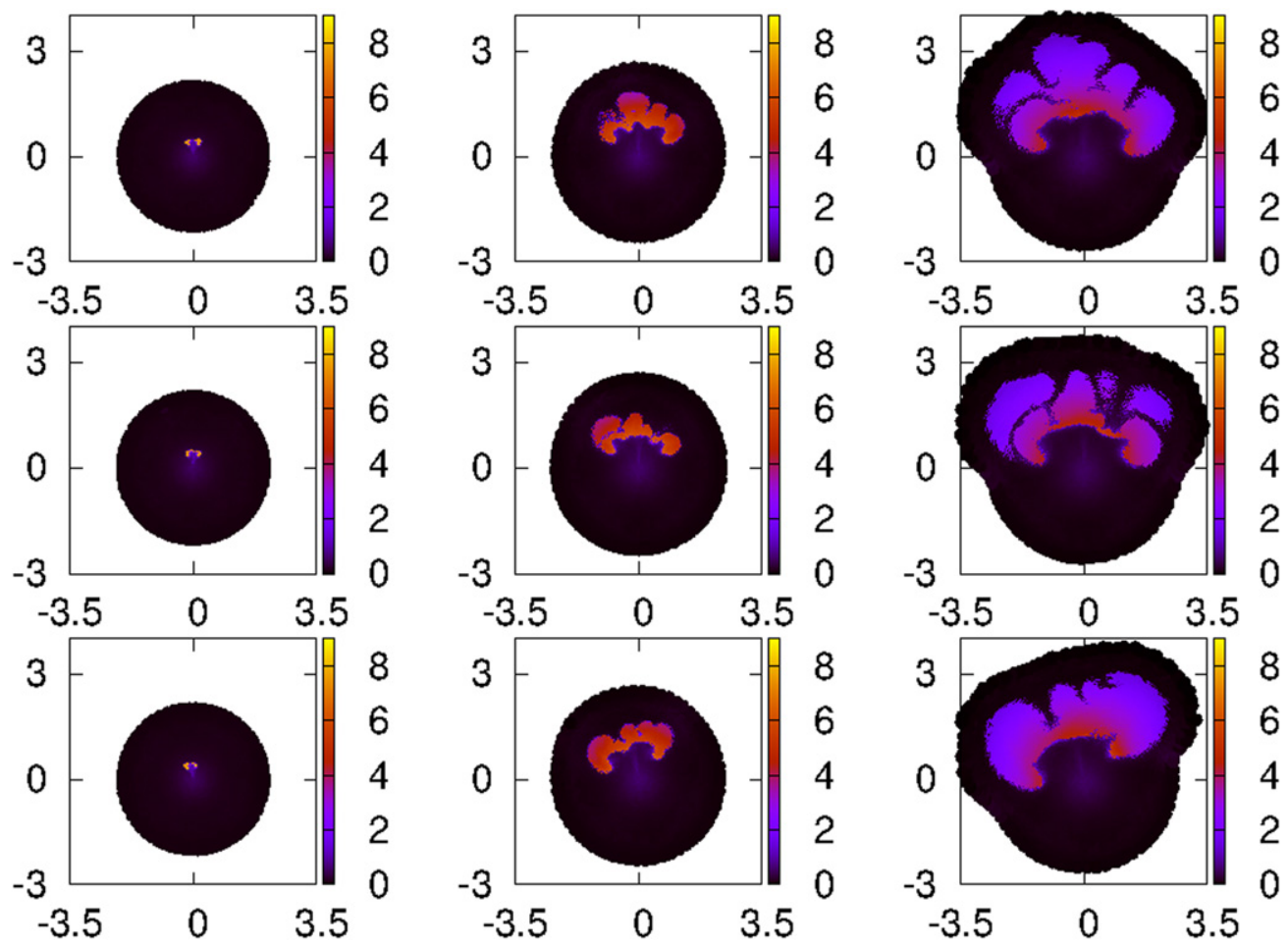

Figure 2. YZ-slice showing a color map of temperature (in units of $10^{9} \mathrm{~K}$ ) for models A (upper row), B (central row), and C (lower row) in Table 1 at times $t=0.59 \mathrm{~s}, t=0.97 \mathrm{~s}$, and $t=1.18 \mathrm{~s}$, respectively. Coordinates are labeled in units of $10^{8} \mathrm{~cm}$.

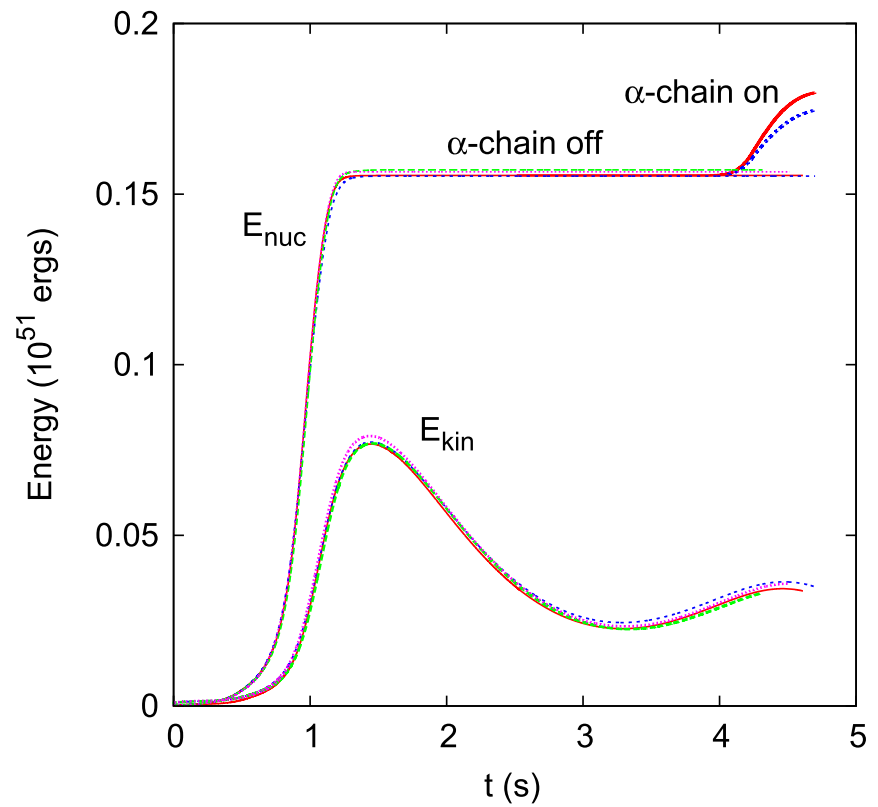

Figure 3. Evolution of the kinetic energy and the released nuclear energy for models A (continuum red line), B (long-dashed line in green), C (dashed-blue line), and $\mathrm{D}$ (dots in pink). After $t=2.5 \mathrm{~s}$, nuclear reactions were either switched off or kept switched on (thick lines).

with similar initial conditions (Röpke et al. 2007; Fink et al. 2014).

The stages that precede the collision of the ashes for models $\mathrm{A}, \mathrm{B}$, and $\mathrm{C}$ are shown in Figure 4. For the reference calculation without rotation (model A), the convergence of ashes takes place around the second snapshot at time $t=3.43 \mathrm{~s}$. As we can see, despite the imprint of hydrodynamic instabilities there is an astonishing symmetry around the line joining the center of the remnant with the meeting point. The collision of the conical high-velocity stream of gas promotes the birth of two jets moving to opposite directions (see the central panels in Figure 4). The basic physics of jets born from the collision of fluid streams in both astrophysics (TenorioTagle et al. 1988) and terrestrial laboratories (Harlow \& Pracht 1966) is well known. Depending on the angle of the collision, it may result in one (for low incident angles) or two jets moving in opposite directions. The latter is just what was reported in the simulations by Plewa (2007) and Meakin et al. (2009) and can also be seen in the second and third columns of Figure 4.

According to Table 1 the amount of ${ }^{56} \mathrm{Ni}$ synthesized during the deflagration is around $0.09 M_{\odot}$ with released nuclear energies $\simeq 0.15 \times 10^{51} \mathrm{erg}$. Thus, in those models where the igniting sparks are localized in a tiny, pointlike region, a transition to a detonation (by the GCD or the PRD mechanisms) is necessary to raise the ${ }^{56} \mathrm{Ni}$ yields to standard observed values, $\simeq 0.6 M_{\odot}$. At this point it is worth noting that the analysis of the radioactive isotopes present in the debris of SN2014J suggests the existence of a small amount of ${ }^{56} \mathrm{Ni}$ in the external layers of the exploding star (Diehl et al. 2015). Such early ejection could be spread in a truncated conical ring made of $\simeq 0.05 M_{\odot}$ of ${ }^{56} \mathrm{Ni}$, detached from the main body of the ejecta (Isern et al. 2016). That amount of ${ }^{56} \mathrm{Ni}$ is curiously close to those shown in Table 1, which were produced during the deflagration of the WD. Although in our models the ejected ${ }^{56} \mathrm{Ni}$ is not exactly inside a truncated cone its distribution is biased toward the hemisphere where the ignition of the WD starts.

The evolution of the rotating models $\mathrm{B}, \mathrm{C}$, and D primarily depends on the angle between the rotational axis and the initial line of flotation of the bubble. When that angle is zero the 

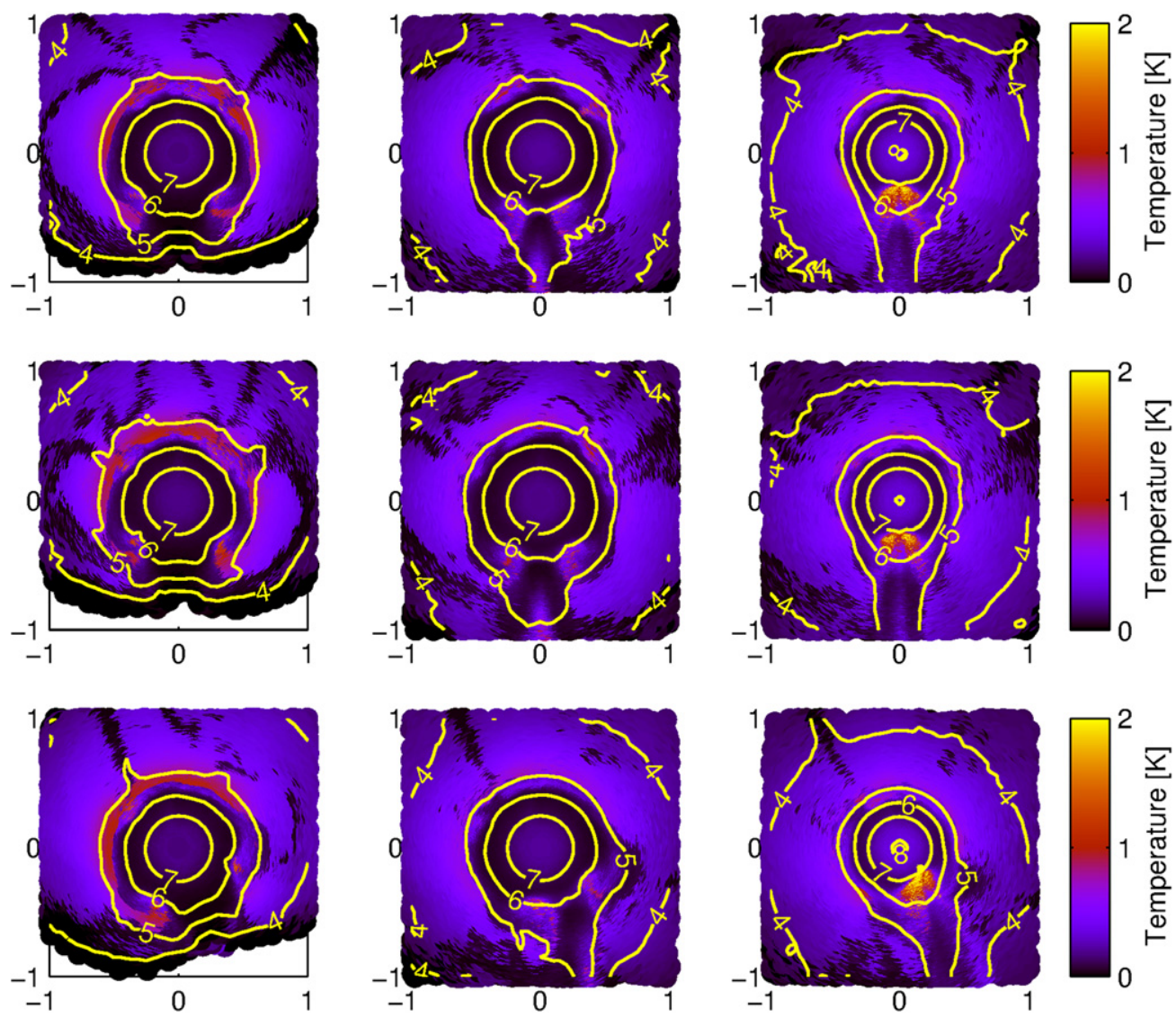

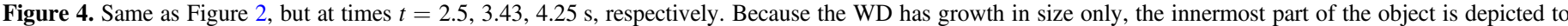
highlight the convergence of the ashes toward the antipodes. Axes are in units of $10^{9} \mathrm{~cm}$.

impact on the dynamics is small because (1) during the first tenths of seconds the bubble rises vertically, therefore the angle between $v_{b}$ and $\Omega$ is small and so is the Coriolis acceleration, and (2) $a_{\mathrm{Col}}$ remains axisymmetric during the lateral expansion of the ashes preserving the symmetry of the flux. Thus, the evolution of model B is similar to that of model A, as can be seen in the central row of panels in Figures 2 and 4. There are minor differences between models $\mathrm{A}$ and $\mathrm{B}$, such as, for example, the lower value of density achieved in model $\mathrm{B}$ at the head of the jet when a fiducial temperature, f.e. $T=3 \times 10^{9} \mathrm{~K}$ $\left(T_{9}=3\right.$ hereafter), is crossed (column 10 in Table 1). Also, the elapsed time needed to account for the first time that temperature is larger for model B (column 11 in Table 1).

Model $\mathrm{C}$ has the same initial angular velocity and kinetic rotational energy as model B but with $\Omega=\Omega_{x}$ going in an orthogonal direction to the initial displacement of the bubble. The overall view of the evolution of model $\mathrm{C}$ is shown in the third row of Figures 2 and 4. We clearly see that the mushroomlike structures are also rotating as they are dragged by the substrate. Actually they would look similar to those of models $\mathrm{A}$ and $\mathrm{B}$ in the comoving rotation frame. The impact of rotation becomes less acute at late times because of the expansion of the WD forces $\Omega$ to drop in order to preserve angular momentum, and by $t \geqslant 1.5 \mathrm{~s}$, any angular movement has practically ceased while the radial expansion is strong. The last two panels of Figure 4 depict the jet formation for this case. We see that one of the effects of the rotation is to blur the component of the jet moving outward whose direction is now less defined. Interestingly, the chunk of the jet moving inward appears to move eccentrically with respect to the line defined by the head of the jet and the center of the remnant. As discussed below this behavior is confirmed by a close-up inspection around the jet region. Despite delivering the same amount of nuclear energy as in cases $\mathrm{A}$ and $\mathrm{B}$, model $\mathrm{C}$ shows some peculiarities at the time of the collision of the ashes. According to Figure 5, the density of the fuel particle with maximum temperature at the jet region is lower than in the nonrotating case. At the fiducial temperature of $T_{\max }=3 \times 10^{9} \mathrm{~K}$ there is a $35 \%$ reduction in density (column 10 in Table 1) and the elapsed time for achieving $T_{\max }$ is a bit shorter. As the conditions to form a detonation by the induction mechanism strongly depend on the density of the fuel (Niemeyer \& Woosley 1997; Dursi \& Timmes 2006), such reduction in density may have consequences for the further evolution of the WD.

Model D was calculated assuming that $\Omega_{x}(r)$ follows the profile given by Equation (1). In this case, the core is rotating as a rigid body with $\Omega_{0}=0.6 \mathrm{~s}^{-1}$ until a radius of $R_{c}=500 \mathrm{~km}$. Beyond $R_{c}$ a linear decay of $\Omega_{x}$ was assumed until it becomes negligible at the surface of the WD. These values of $\Omega_{0}$ and $R_{c}$ were chosen so that the rotational energy of model $\mathrm{D}$ at $t=0 \mathrm{~s}$ is almost the same as that of models $\mathrm{B}$ and C. The evolution of model D, summarized in Figure 6, was not very different from that of model $\mathrm{C}$, as the amount of nuclear energy released during the deflagration is nearly the same. For this model, the value of density at the head of the jet, when the temperature $T_{9}=3$ is achieved for first time in the colliding region, is larger than in model $\mathrm{C}$ but lower than in model $\mathrm{A}$ (see 


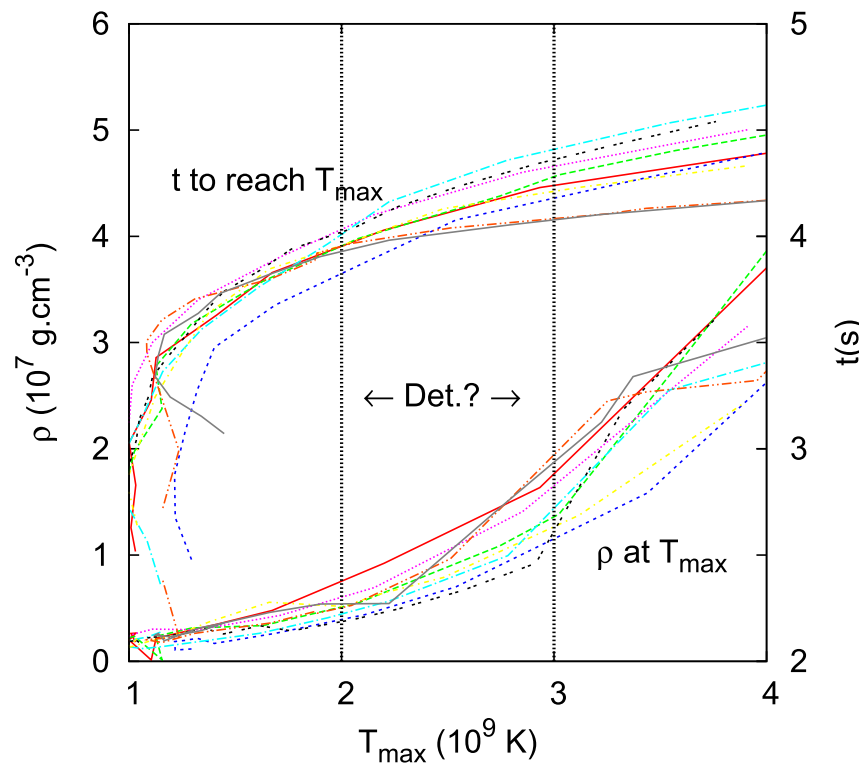

Figure 5. Density of the particle as a function of the instantaneous maximum value of the temperature of fuel particles $T_{\max }$ achieved in the collision region (with nuclear reactions turned off). Model A (continuum line in red), model B (dashed line in green), model C (short-dashed line in blue), model D (dots in pink), model E (dashed-dotted line in light blue), model $\mathrm{F}$ (dashed line in yellow), model $\mathrm{G}$ (two-points in black), model $\mathrm{H}$ (dashed-dotted line in orange), and model I (short lines in brown). The elapsed time needed to reach $T_{\max }$ (uppermost curves) can be read on the vertical axis at the right. The vertical bars in black show the region where the transition to a detonation may occur.
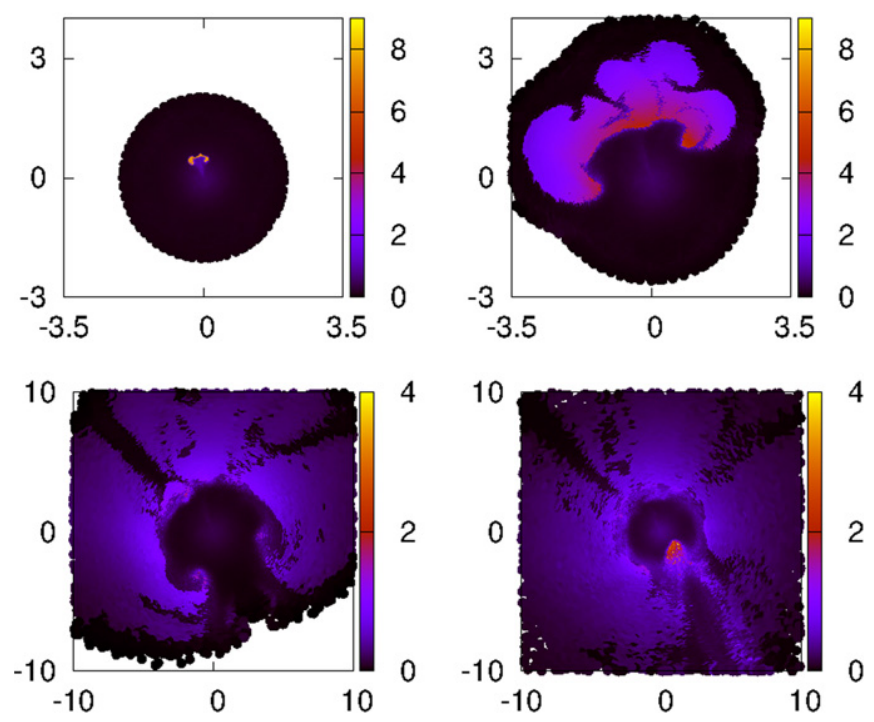

Figure 6. Same as Figures 2 and 4 but for model D at times $t=0.6,1.21 \mathrm{~s}$ (upper rows) and $t=2.54,4.25 \mathrm{~s}$ (lower rows).

Table 1). These results support the common idea that the total amount of released nuclear energy is the primary parameter that controls the thermodynamic conditions in the colliding region, which in turn determines the chances for the detonation of the core. Nevertheless, rotation also plays a role because as shown below the symmetry of the convergence of the ashes is also broken in model D. As in model $\mathrm{C}$, the jet is digging tangentially into the core, thus changing the environmental conditions at the tip of the jet.

Finally, Figure 7 (models A and B) and Figure 8 (models C and D) show a close-up view of the jet region at the time when
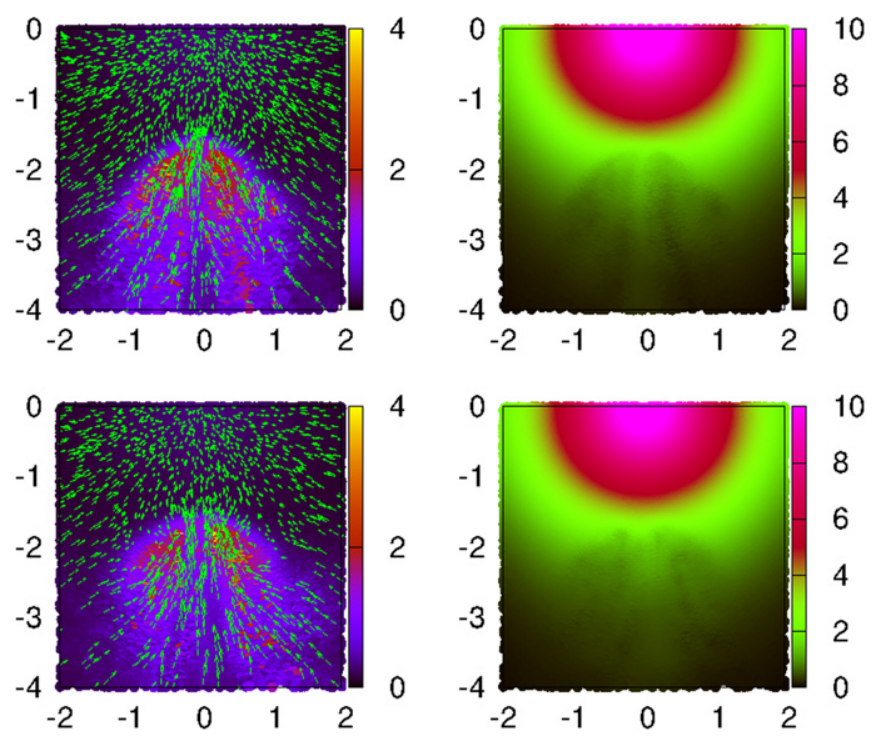

Figure 7. Detail of the collision region for model A at time $t=4.229 \mathrm{~s}$ (upper panels) and model B at $t=4.289 \mathrm{~s}$ (lower panels). The left column shows the velocity field superimposed on the temperature (in units of $10^{9} \mathrm{~K}$ ) color map. The right column depicts density in units of $10^{7} \mathrm{~g} \mathrm{~cm}^{-3}$.
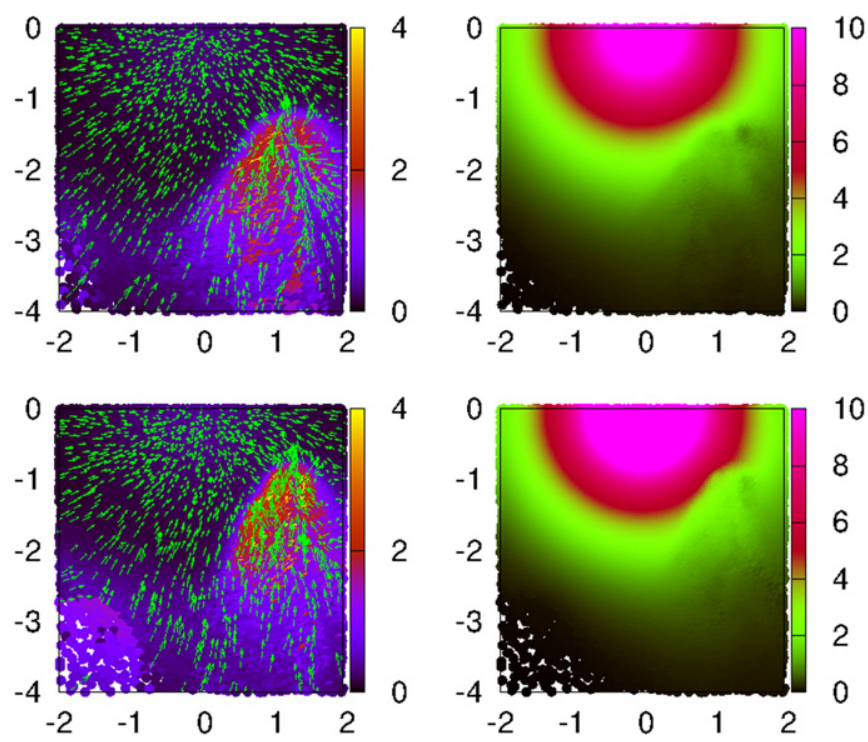

Figure 8. Same as Figure 7 but for models $\mathrm{C}$ and D at times $t=4.277 \mathrm{~s}$ and $t=4.25 \mathrm{~s}$.

a detonation could have emerged if nuclear reactions were switched on. In all cases we can see the jet traveling toward the core of the remnant. In Figure 7 we see that the jet is broad $(\geqslant 1000 \mathrm{~km})$ due to the low resolution in this diluted region. The jet is accreting burned material at its sides through a steady accretion shock and the pressure inside the jet is roughly balanced by the ram pressure of the infalling material. The detailed structure of the jet has been described in Meakin et al. (2009) and Seitenzahl et al. (2009a) using a high-resolution axisymmetric approach. In models A and B the velocity field inside the jet is pointing to the center of the remnant. In these models, the jet is digging into the core (see the rightmost panels in Figure 7), whereas at the tip of the jet there is a compressed region with higher temperature and density, and is made of fuel. According to previous studies it is just in this region where there is some chance for a detonation (Seitenzahl et al. 
2009a) but knowing it unambiguously would require a 3D calculation with very high resolution $(\leqslant 500 \mathrm{~m})$ that incorporates many pieces of physics (rotation among them). Models $\mathrm{C}$ and $\mathrm{D}$ rotating around a tilted axis show, however, a different behavior. According to Figure 8, a jet is also born in the region of the collision of the ashes, but now the focusing effect is weaker and, more important, the velocity field inside the jet is not pointing to the center of the core. This effect can also be seen in the color map of density where the jet is "biting" the core laterally, which changes the density conditions found by precursor shock ahead of the tip of the jet. Therefore, it seems that even a moderate amount of rotation may have an impact on the detonation formation and future studies should include this ingredient in the simulations.

\subsection{Resolution Study}

To investigate the sensitivity of the results with the adopted resolution we carried out five additional simulations: models $\mathrm{E}$, $\mathrm{F}$, and $\mathrm{G}$, using $4 \mathrm{Mp}$, and models $\mathrm{H}$ and $\mathrm{I}$ with $8 \mathrm{Mp}$ (Table 1). A caveat is necessary here because changing the number of particles demands new initial models. Due to the Mont-Carlo-like nature of the SPH technique, it is impossible to have identical stable initial models with the same mass, central density, and radius but different particle number. Moreover, as noted in Section 2, building a stable WD with a total mass approaching the Chandrasekhar mass limit is a task that requires not only patience but also a computational effort that can be as high as the actual calculation of the explosion itself. Thus, our initial models with 2,4 , and $8 \mathrm{Mp}$ are just different SPH realizations of the same WD with total mass $M=1.376 M_{\odot}$. In this sense, their structure and stability properties are not $100 \%$ identical. This has to be taken into account when interpreting the results.

Model E, calculated with $4 \mathrm{Mp}$, leads to a slightly stronger explosion than model A, calculated with $2 \mathrm{Mp}$. Both are norotating models so the differences mainly come from the different resolution. The released nuclear energy was $\simeq+7 \%$, larger in the high-resolution model. Because the nuclear energy input is the primary parameter driving the explosion, the remaining magnitudes shown in Table 1 do consistently follow that trend. In particular, the minimum value of the central density is lower in model $\mathrm{E}$ than in model $\mathrm{A}$, as well as that of the density at the top of the jet when the fiducial temperature $T_{9}=3$ is achieved (as in models $\mathrm{A}, \mathrm{B}, \mathrm{C}$, and D nuclear reactions were also switched off during the collision of the ashes in models E, F, G, H, and I).

Model F, calculated with $4 \mathrm{Mp}$, spins around the $\mathrm{X}$-axis with the same rotational velocity as model $\mathrm{C}$, calculated with $2 \mathrm{Mp}$. In this case the nuclear energy released during the explosion of model $\mathrm{F}$ is slightly lower than that of model $\mathrm{C}$ (Table 1). Although the difference is small, $\simeq-3 \%$, it is a little puzzling because in the case of the non-rotating models, $\mathrm{E}$ and $\mathrm{A}$, the difference amounts to $+7 \%$. We suspect that such behavior is due to the interplay of rotation with subtle differences among the initial models (such as stability or the residual numerical noise). Because of the lower amount of released energy, the minimum value of the central density is larger in model $\mathrm{F}$, $\rho_{c}(\min )=4.8 \times 10^{7} \mathrm{~g} \mathrm{~cm}^{-3}$, than in model $\mathrm{E}$, $\rho_{c}(\min )=3.9 \times 10^{7} \mathrm{~g} \mathrm{~cm}^{-3}$, and consistent with model $\mathrm{C}$, $\rho_{c}(\min )=4.7 \times 10^{7} \mathrm{~g} \mathrm{~cm}^{-3}$. The density at the top of the jet when $T_{9}=3\left(\rho_{\text {jet }}=1.27 \times 10^{7} \mathrm{~g} \mathrm{~cm}^{-3}\right)$ in model $\mathrm{F}$ is also consistent with that of model $\mathrm{C}\left(\rho_{\text {jet }}=1.16 \times 10^{7} \mathrm{~g} \mathrm{~cm}^{-3}\right)$ in

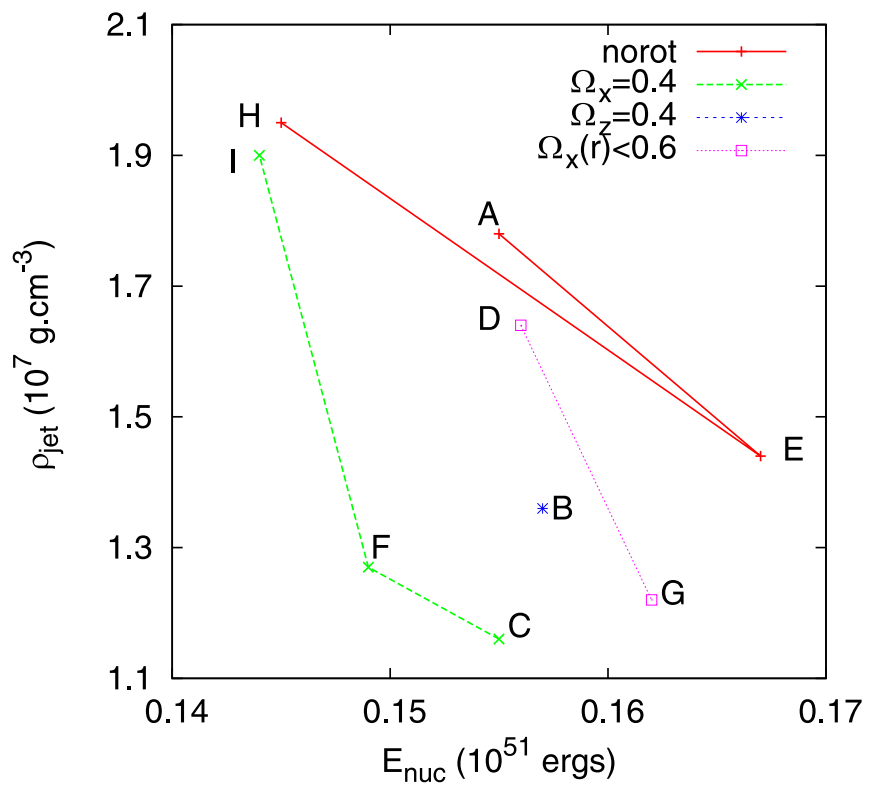

Figure 9. Location of models in Table 1 along the diagram $\left(\rho_{\text {jet }}-E_{\text {nuc }}\right)$ when the temperature of an unburned particle in the colliding region exceeds 3 billions degrees.

light of the differences in the released energy (Table 1 and Figure 5). The best resolved models, $\mathrm{H}$ and I, calculated with $8 \mathrm{Mp}$, released a bit less nuclear energy and gave a comparatively higher density at the top of the jet. Unlike the rest of the cases, the difference in $\rho_{T_{9}=3}^{\text {jet }}$ between models $\mathrm{H}$ and $\mathrm{I}$ is now much less pronounced. Thus, we do not strictly see the convergence of the models with increasing number of particles but rather some scatter of the results around $E_{\text {nuc }} \simeq 0.15510^{51} \mathrm{erg}$. A comparison among all calculated models is shown in Figure 9, which represents the density of the fuel on top of the jet when $T_{9}=3$ as a function of the released nuclear energy. As we can see all models settle in a band centered at $\simeq 0.15510^{51}$ erg with a dispersion less than $\simeq 7 \%$. Thus, Figure 9 roughly reflects the dependence of the results with respect to the initial model, resolution, and the inclusion (or not) of a moderate amount of rotation, respectively. Although, as commented above, the peculiarity of the SPH initial models make it difficult to distinguish among these three items, the distribution of models along the figure suggests: (a) models with larger nuclear combustion do systematically lead to lower values of density on top of the colliding region, and (b) at a constant released nuclear energy, rotation reduces the value of the density at the jet location.

On another note, the qualitative behavior of models $\mathrm{C}, \mathrm{D}, \mathrm{F}$, $\mathrm{G}$, and I during the convergence of the ashes at the antipodes is similar as the collision also takes place obliquely in the rotating models calculated with increased resolution. As a conclusion, we may say that the higher resolution models behave similarly to the reference models calculated with $2 \mathrm{Mp}$ and described in the precedent section. An increase in the number of particles may lead to slight variations in the released nuclear energy but, as shown in Figure 5, the density at the top of the jet during the collision of the nuclear ashes, when $T_{9} \simeq 3$, remains systematically lower in rotating models. 

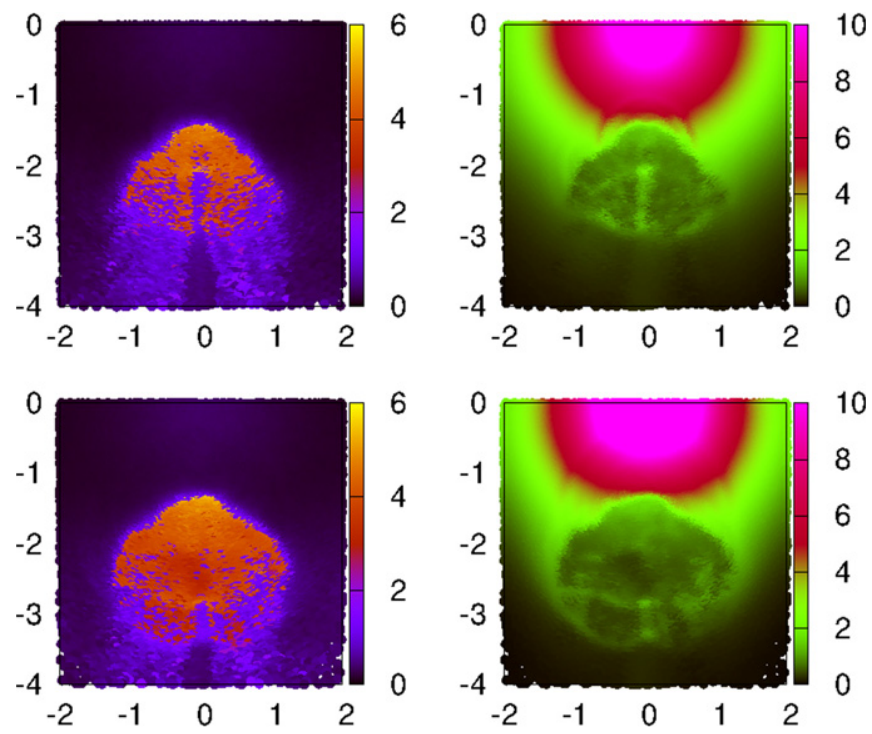

Figure 10. Same as Figure 7 but for model A at times $t=4.28032 \mathrm{~s}$ and $t=4.41304 \mathrm{~s}$ with the nuclear reactions turned on.

\subsection{Core Detonation?}

The initiation of the detonation at the core edge, induced by the inwardly moving jet, has been analyzed with some detail by Seitenzahl et al. (2009a) assuming that the jet is axisymmetric and restricting the calculation to a small region around the symmetry axis at the core interface. Their convergence study, with resolution between $\simeq 4$ and $\simeq 0.1 \mathrm{~km}$, showed that in some cases a detonation is formed via the gradient mechanism when a given volume of fuel has a shallow enough density and temperature distribution that it burns in a time lower than the sound crossing time. A detonation occurs provided that the size of the burned zone is larger than a critical value that strongly depends on density. According to Röpke et al. (2007) that radius is $\simeq 120 \mathrm{~km}$ at $\rho=3 \times 10^{6} \mathrm{~g} \mathrm{~cm}^{-3}$ and $T_{9}=2.3$, but it decreases to $\simeq 8 \mathrm{~km}$ at $\rho=10^{7} \mathrm{~g} \mathrm{~cm}^{-3}$ and $T_{9}=2.2$. In the calculations by Seitenzahl et al. (2009a), with a resolution of $0.5 \mathrm{~km}$, these criteria are satisfied behind a compression front that moves transversely to the tip of the jet, facilitating the initiation of the detonation.

Even though our resolution $(\simeq 25-70 \mathrm{~km}$, between the center of the core and the top of the jet) is not enough to elucidate if there is a detonation of the core ${ }^{5}$, we have followed the penetration of the jet farther to compare the cases with and without rotation. Starting at some point at the plateau in Figure 3, we resume the calculation, switching on the nuclear reactions. In the same figure we see that the nuclear energy input becomes relevant again after $t=3.95 \mathrm{~s}$ for model $\mathrm{A}$ and $t=3.97 \mathrm{~s}$ for model C. In Figure 10 we show the color map of temperature and density for model A at times $t_{1}=4.28032 \mathrm{~s}$ and $t_{2}=4.41304 \mathrm{~s}$. It can be seen that the size of the hightemperature region grows in time as matter flows into the hot volume through the accretion shock. Nevertheless, the penetration of the apex of the jet in $\Delta t=t_{2}-t_{1}$ is hardly perceptible. On the top of the conical jet, but detached from it, there is a weak shock wave digging into the core. That wave is

\footnotetext{
5 According to the detonation criteria set in Seitenzahl et al. (2009b) the transition to a detonation is not ruled out in any of the models shown in Table 1. Nevertheless, rotational models appear to be the less favored to undergo a detonation.
}

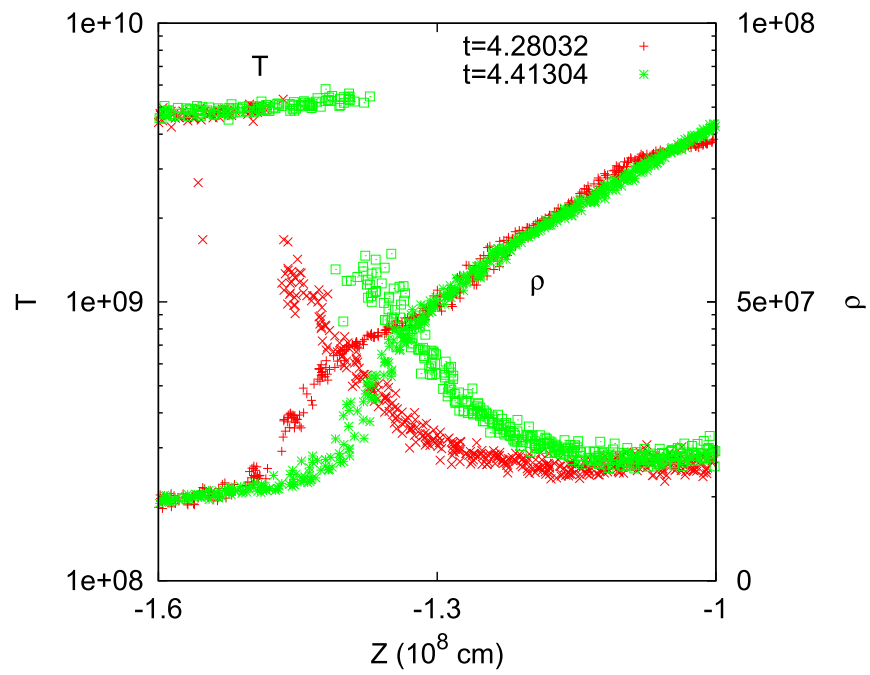

Figure 11. Profiles of temperature and density of model A obtained from a onedimensional cut around the symmetry line of the jet at times $t=4.28032 \mathrm{~s}$ and $t=4.41304 \mathrm{~s}$
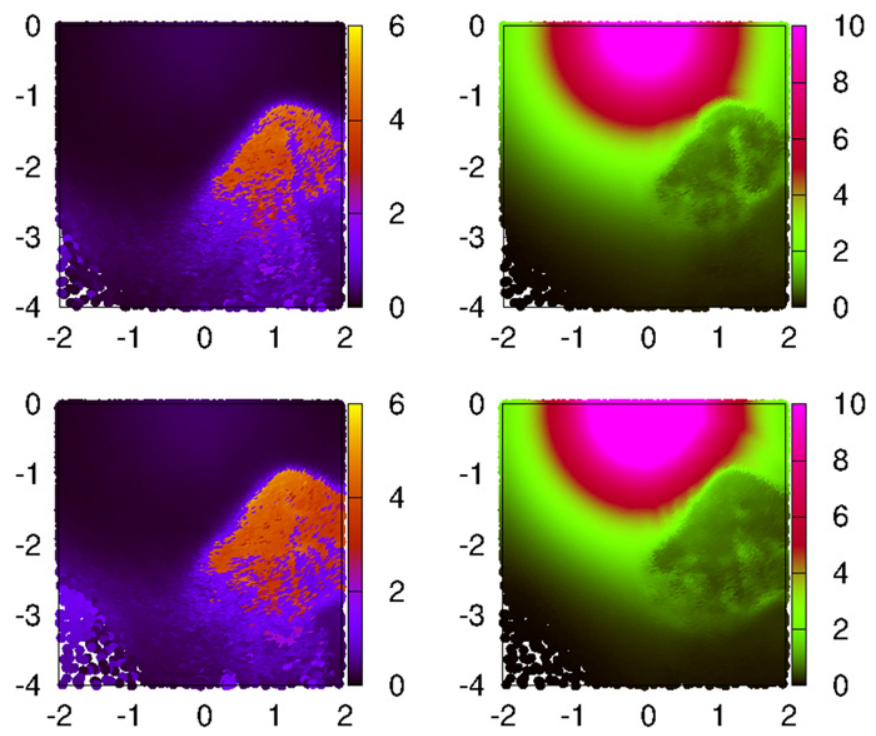

Figure 12. Same as Figure 7 but for model $\mathrm{C}$ at times $t=4.28205 \mathrm{~s}$ and $t=4.40775 \mathrm{~s}$ with the nuclear reactions turned on.

clearly visible in the density maps of Figure 10, especially at $t_{1}=4.28032 \mathrm{~s}$. A one-dimensional cut around the symmetry axis showing the density and temperature profiles as a function of coordinate $Z$ is shown in Figure 11. These profiles are moving with phase velocity $\leqslant 1000 \mathrm{~km} \mathrm{~s}^{-1}$, lower than the sound speed in the unburned material, $C_{s} \simeq 4000 \mathrm{~km} \mathrm{~s}^{-1}$. Thus, at our last computed time, $t_{2}=4.41304 \mathrm{~s}$, there was not a clear signal of detonation in model $\mathrm{A}$.

In Figure 12 are depicted the temperature and density color maps of model $\mathrm{C}$ at times $t_{1}=4.28205 \mathrm{~s}$ and $t_{2}=4.40775 \mathrm{~s}$. As in Model A the nuclear reactions were turned on above $t=2.5 \mathrm{~s}$ and the color map focuses on the region around the jet. The head of the jet is penetrating obliquely into the core while it grows in size owing to the nuclear combustion. As in model A a precursor shock is formed on top of the jet at time $t_{1}$. This weak shock soon detaches from the subsonic jet and moves right into the core, distorting its spherical symmetry. Again there was not any clear indication of detonation in this 
region. The evolution of the released nuclear energy of models $\mathrm{A}$ and $\mathrm{C}$ at late times is shown in Figure 3 (thick red and blue lines, respectively). As we can see both curves tends to saturate but the slope of the released nuclear energy is lower in model $\mathrm{C}$. This is in agreement with the information shown in Figure 5 concerning the density of the fuel at a given temperature in the collision region. That density is lower in the rotating models and so are the nuclear reaction rate and the released nuclear energy.

If a detonation does not occur there are two possible outcomes for the remnant: (1) it simply remains oscillating while accreting a fraction of the ejected material made for the most part of unburned particles, or (2) the fallback onto the core of some of the previously expelled matter produces an accretion shock. As the infalling material crosses the accretion shock it is compressed and heated, leading to a second chance for a detonation of the core. This is the PRD explosion mechanism postulated by Bravo \& García-Senz (2006). We have tracked the evolution of models $\mathrm{A}$ and $\mathrm{C}$ until $t \simeq 12 \mathrm{~s}$. In both cases the evolution is quite complex showing several episodes of ash collision while the core is oscillating. The follow-up of this long phase, probably leading to the PRD ignition of the core, is left for a future work.

\section{DISCUSSION AND CONCLUSIONS}

Multidimensional simulations of the thermonuclear explosion of WDs have, for the most part, assumed that such events take place in a non-rotating progenitor. This is actually a wrong hypothesis because the same accretion process that builds up the mass of the WD also transfers angular momentum from the disk to the compact object. As a result the WD could be spinning at angular velocities as high as $\Omega \simeq 2-3 \mathrm{~s}^{-1}$ at the moment of ignition (Piersanti et al. 2003; Yoon \& Langer 2005; Domínguez et al. 2006). The reduction in the effective gravity caused by the centrifugal force changes the structure of the progenitor prior the explosion. The density profile is no longer spherically symmetric and, for the same central density, rotating WDs could be more massive than their non-rotating counterparts. If the amount of rotation is low, to handle the explosion without the intervention of rotation is usually a good approximation. But in some particular scenarios even a slow rotation may have consequences for the development of the explosion. This is the case in the GCD scenario which relies on the efficiency of the convergence of the nuclear flame at the antipodes of the ignition point. A good efficiency is achieved provided the deflagration remains axisymmetric with respect to the line defined by the center of mass of the WD and the ignition point. Nonetheless, even a small amount of rotation may break the symmetry of the process because the Coriolis force is by nature non-axisymmetric and, being proportional to the velocity of the hot rising blobs, it can be strong. The Coriolis force is also acting in deflagrations arising from a multipoint ignition but in that case the stochasticity of the ignition may render its final impact less relevant.

In this work we have studied the impact of having a moderate amount of rotation in the fate of the deflagration arising from a point-like ignition using the Coriolis force. Considering angular velocities of $\Omega \simeq 0.4 \mathrm{~s}^{-1}$ is not at odds with the hypothesis of spherical symmetry and allows a meaningful comparison of the results between rotating and non-rotating models. Such angular velocity is small enough to not change the density profile and equilibrium properties of the progenitor, but sufficient to appreciably push the hot blobs of incinerated material as they rise at velocities approaching the local sound speed. A larger value of $\Omega$ will make the Coriolis force even stronger and, in this sense, our calculations are rather conservative. On another note, moderate spinning WDs could be less rare. Exploding WDs rotating with $\Omega \leqslant 1 \mathrm{~s}^{-1}$ are expected for compact systems with total masses close to the canonical Chandrasekhar mass limit. The physical structure of these WDs would be similar to that of their non-rotating counterparts (Piersanti et al. 2003; Domínguez et al. 2006), and as stated in the introduction, the central deflagration of a fast spinning WD leaves a large amount of unburned matter, which is incompatible with observations (Pfannes et al. 2010b).

Our results show that the effect of rotation is more pronounced when the rotation axis is orthogonal to the ascending line of the bubble, reinforcing the idea that the Coriolis force is the main agent behind the differences between spinning and non-spinning models. In this respect, it is worth mentioning that the value of the angle between the rotation axis and the ascending line of the bubble is unknown. In order to have better insight of realistic values of this angle, detailed 3D calculations of the progenitor including rotation are needed.

For a similar released nuclear energy, models with low to moderate rotation show two distinctive features (see Table 1 and Figure 9): (1) after the convergence of the deflagration at the antipodes, the density of an unburned particle when $T_{9} \simeq 3$ is lower in rotating models and (2) as a result of the collision of the ashes, a conical jet is born which grows in size through an accretion shock. In non-rotating models the jet is axisymmetric with its apex heading right to the center of the core. In rotating models, however, the conical symmetry is not so perfect and the jet is no longer pointing to the center of the core. Both features suggest that, along with the total released nuclear energy, rotation is also an important parameter to take into account to understand the GCD route to SNe Ia.

Several studies have stated that the self-consistent detonation of the core of a WD by the GCD mechanism is not easy, probably requiring a fine tuning of the physical conditions at the convergence region of the flame. In particular the released nuclear energy during the deflagration phase has to be low (Röpke et al. 2007), the geometry of the flame at late times should remain as axisymmetric as possible to also produce an axisymmetric jet (Meakin et al. 2009), and finally, giving birth to a detonation requires a precise preconditioning of the plasma at the tip of the jet (shallow gradients of $\rho, T$ in a large enough volume; Seitenzahl et al. 2009a). The resolution achieved in the models described in Table 1 is only moderate, thus precluding any firm conclusion on the deflagration to detonation transition. Our exploratory calculations using low to moderate rotators suggest that detonating the core of the WD via the GCD mechanism could be even more difficult in spinning models because the symmetry of the deflagration is broken by the Coriolis force. Therefore a deeper understanding of the rotational features of a massive WD on the verge of explosion is needed.

This work has been supported by the MINECO-FEDER Spanish projects AYA2013-42762-P and AYA2014-59084-P and by the AGAUR (D. García-Senz) and AYA2011-22460 (I. Domínguez). It has also been supported by the Swiss Platform for Advanced Scientific Computing (PASC) project DIAPHANE (R. Cabezón) and by the European Research Council 
(FP7) under ERC Advanced Grant Agreement No. 321263FISH. (R. Cabezón and F.K. Thielemann). The Basel group is a member of the COST Action New CompStar. The authors acknowledge the support of sciCORE (http://scicore.unibas. $\mathrm{ch} /$ ) scientific computing core facility at University of Basel, where some of the calculations were performed. D. GarcíaSenz dedicates this work to the memory of his father, Francisco García Serrano, who passed away recently.

\section{APPENDIX \\ FLAME IMPLEMENTATION}

Nuclear flames were handled using a reaction-diffusion scheme. Therefore, the actual thickness of the flame was artificially enlarged to the spatial local resolution of the simulation, roughly the smoothing length parameter $h$. The actual conductivity coefficient was conveniently re-scaled to obtain a prescribed velocity for the flame, which was in turn computed from a flame subgrid model. Those cases where the scaling factor is one correspond to real microscopic laminar flames. The evolution of the flame is basically controlled by the energy equation:

$$
\frac{d u}{d t}=\frac{1}{\rho^{2}} P \frac{d \rho}{d t}+\frac{1}{\rho} \nabla \cdot(\kappa \nabla T)+\dot{S}_{\text {nuc }}
$$

where $\kappa$ is the thermal conductivity, $P$ is the pressure, and $u$ is the specific internal energy. Now consider the following linear transformations:

$$
\boldsymbol{r} \longrightarrow \boldsymbol{r}^{\prime}=a \boldsymbol{r} ; \quad t \longrightarrow t^{\prime}=b \quad t
$$

which maps the coordinate $\{r, t\}$ framework of the microscopic thermal wave to the laboratory $\left\{r^{\prime}, t^{\prime}\right\}$ framework of the hydrocode. Equation (2) can be written as:

$$
\begin{aligned}
d u= & \frac{1}{\rho^{2}} P d \rho+a^{2} \frac{1}{\rho} \nabla^{\prime} \cdot\left(\kappa \nabla^{\prime} T\right)\left(\frac{d t^{\prime}}{b}\right) \\
& +\dot{S}_{\text {nuc }}\left(\frac{d t^{\prime}}{b}\right)
\end{aligned}
$$

On the other hand, for a steady conductive flame moving with velocity $\boldsymbol{v}_{c}$ :

$$
\boldsymbol{v}_{c} \cdot \boldsymbol{\nabla}=\frac{\partial}{\partial t}
$$

Using the scaling transformations (3) in Equation (5) gives:

$$
a \boldsymbol{v}_{c} \cdot \nabla^{\prime}=b \frac{\partial}{\partial t^{\prime}}
$$

Therefore, only for $a=b$ will the scaled flame move just at the conductive velocity $v_{c}$. Thus, setting the ratio $a / b$ to:

$$
\frac{a}{b}=\frac{v_{f}}{v_{c}}
$$

leads to a prescribed effective velocity of the flame, $v_{f}$. The practical procedure to implement the flame in the numerical scheme is:

1. Make an estimation of parameter $a=\left(\frac{h}{\delta}\right)$, where $h$ is the smoothing length and $\delta$ is the actual microscopic width of the flame (Timmes \& Woosley 1992).
2. Calculate the effective flame velocity $v_{f}$ using a subgrid model (see below). The parameter $b$ is then set to $b=a\left(\frac{v_{c}}{v_{f}}\right)$, where $v_{c}$ is the laminar flame velocity.

3. Compute the conductivity $\kappa\left(\rho, T, X_{i}\right)$ and released nuclear energy rate and evolve the model using Equation (4) as an energy equation to describe the evolution of the internal energy and temperature.

Because the scheme uses both the physical conductivity and nuclear reaction rates calculated through a 14 isotope network, it will provide the actual conductive velocity in the limit of very large resolution characterized by $a=b \simeq 1$.

The nuclear network is able to follow all stages of the combustion, including the nuclear statistical equilibrium regime. Our network is an $\alpha$-chain linking symmetric nuclei in the range ${ }^{4} \mathrm{He}-{ }^{60} \mathrm{Zn}$ and completed with the binary reactions ${ }^{12} \mathrm{C}+{ }^{12} \mathrm{C},{ }^{16} \mathrm{O}+{ }^{16} \mathrm{O},{ }^{12} \mathrm{C}+{ }^{16} \mathrm{O}$. A specific feature in our calculations is that the integration of the nuclear network is done implicitly with the temperature equation. Solving abundances and temperature simultaneously is important to damp the oscillations which often appear near equilibrium when photodisintegration reactions become important. Nevertheless, in order to save computing time, the material is transformed isochorically to NSE elements once the temperature has risen over 3.5 billion degrees and density is higher than $5 \times 10^{7} \mathrm{~g} \mathrm{~cm}^{-3}$. Our NSE routine incorporates the same amount of nuclear species as the $\alpha$-network, so that both are totally compatible. The jump to the NSE state is handled implicitly with the energy equation, so that the equilibrium abundances and temperature are found simultaneously. Once the self-consistent NSE state is achieved the time-step rises and the evolution is followed again with the 14 isotope network. Such a procedure allows us to adequately follow the freeze out of nuclear reactions.

The flame model described above has been verified using a couple of tests in the line of the verification strategies proposed in Townsley et al. (2007). We basically checked that the flame is moving at a prescribed constant velocity through the WD interior. This is a quite demanding test because the density ahead of the flame changes by two orders of magnitude between the center and the location where the flame dies. Once $v_{f}$ is chosen, the parameters $a=h(\rho) / \delta(\rho)(\delta(\rho)$ is the real microscopic width of the flame) and $b=a\left(v_{c}(\rho) / v_{f}\right)\left(v_{c}\right.$ is the conductive flame velocity) self-adapt to the environmental conditions and no further tuning of the parameters is necessary. To check the flame we have chosen the velocities $v_{f}=100 \mathrm{~km} \mathrm{~s}^{-1}$ and $v_{f}=300 \mathrm{~km} \mathrm{~s}^{-1}$. The former is close to the laminar velocity of the flame at the beginning of the explosion where densities are $\simeq 10^{9} \mathrm{~g} \mathrm{~cm}^{-3}$ and the flame is not yet beset by instabilities. The latter value is representative of the effective flame velocity at more advanced stages when hydrodynamic instabilities and turbulence control the explosion. It is expected that a great deal of combustion takes place in the range $100 \leqslant v_{\text {eff }} \leqslant 300 \mathrm{~km} \mathrm{~s}^{-1}$. The results of the calculations are shown in Figure 13 where the evolution of the burned mass for the two flame velocities above is depicted and compared to the theoretical expectations. As it can be seen, the numerical effective flame velocity gives the correct amount of incinerated mass even at large elapsed times, when $\simeq 1 M_{\odot}$ has been burned, thus giving confidence to the flame propagation algorithm. 


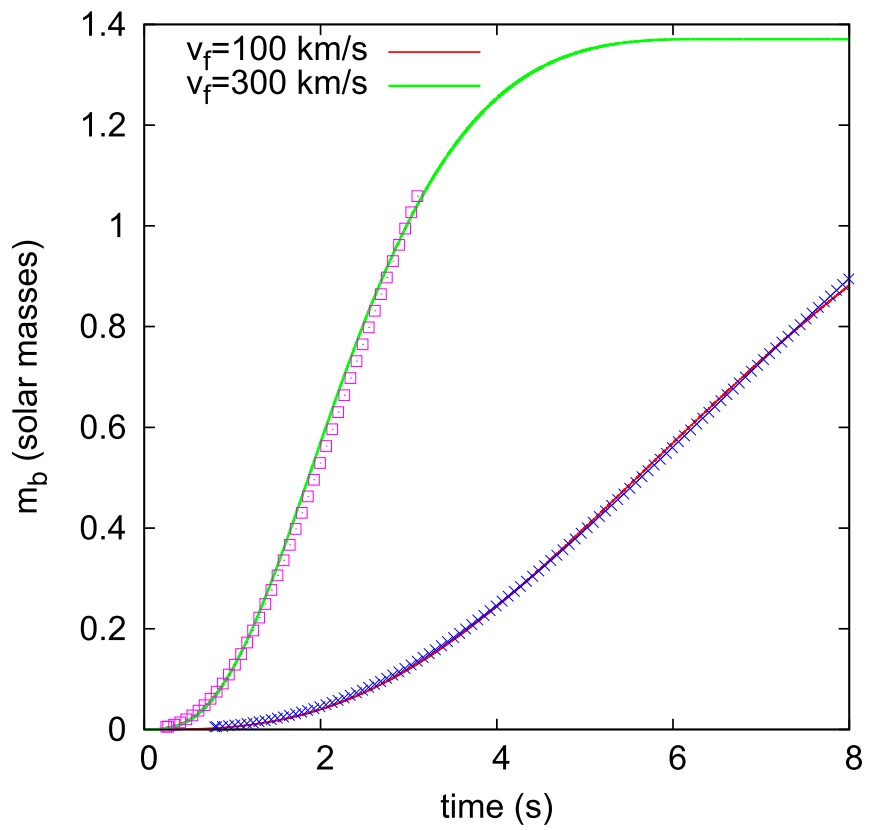

Figure 13. Propagation of a laminar flame moving at constant velocity in the density profile of a massive white dwarf with central density $\rho=2.6 \times 10^{9} \mathrm{~g} \mathrm{~cm}^{-3}$. The burned mass as a function of time is shown for two effective flame velocities $v_{\text {eff }}=100 \mathrm{~km} \mathrm{~s}^{-1}$ (blue crosses) and $v_{\text {eff }}=300 \mathrm{~km} \mathrm{~s}^{-1}$ (pink squares). Continuum lines indicate the analytical evolution.

A subgrid model was built by simply assuming that the size of the velocity fluctuation at the minimum scale length solvable by the hydrocode is just the effective burning velocity at such a scale (Damköhler 1940). In order to evaluate the characteristic turnover velocity of eddies with size $\simeq h$ we assume an isotropic velocity field around the particle. In cylindrical coordinates the velocity at a distance $r$ of a given particle is described by:

$$
\boldsymbol{v}(r, \varphi)=K r^{n} \tau
$$

where $\tau$ is the azimuthal unit vector. For $n=1$ expression (8) reduces to rigid rotation (see Figure 14). Cases with $n=1 / 2$ and $n=1 / 3$ are representative of RT instability and turbulence (Niemeyer \& Woosley 1997).

Taking the curl of the above equation in cylindric coordinates and evaluating it at $r=h$ gives:

$$
\boldsymbol{\nabla} \times v=(n+1) K h^{n-1} \boldsymbol{k}
$$

where $\boldsymbol{k}$ is the unit vector along the $Z$-axis. Constant $K$ in Equation (8) becomes:

$$
K=\frac{1}{n+1} h^{1-n}|\nabla \times v|_{\mathrm{SPH}}
$$

where $|\nabla \times v|_{\text {SPH }}$ stands for the curl calculated with the hydrocode. Therefore a rough estimation of the turnover velocity at scale $h$ is

$$
v(h) \simeq K h^{n}=\frac{1}{n+1} h|\nabla \times v|_{\mathrm{SPH}} .
$$

The turnover velocity $v_{t}$ is finally taken $v_{t}=\alpha v(h)$ with $0<\alpha<1$. In our calculations we use $n=1 / 3$, characteristic of turbulence and $\alpha=0.25$ fitted so that the total released nuclear energy during the failed explosion starting in a non-

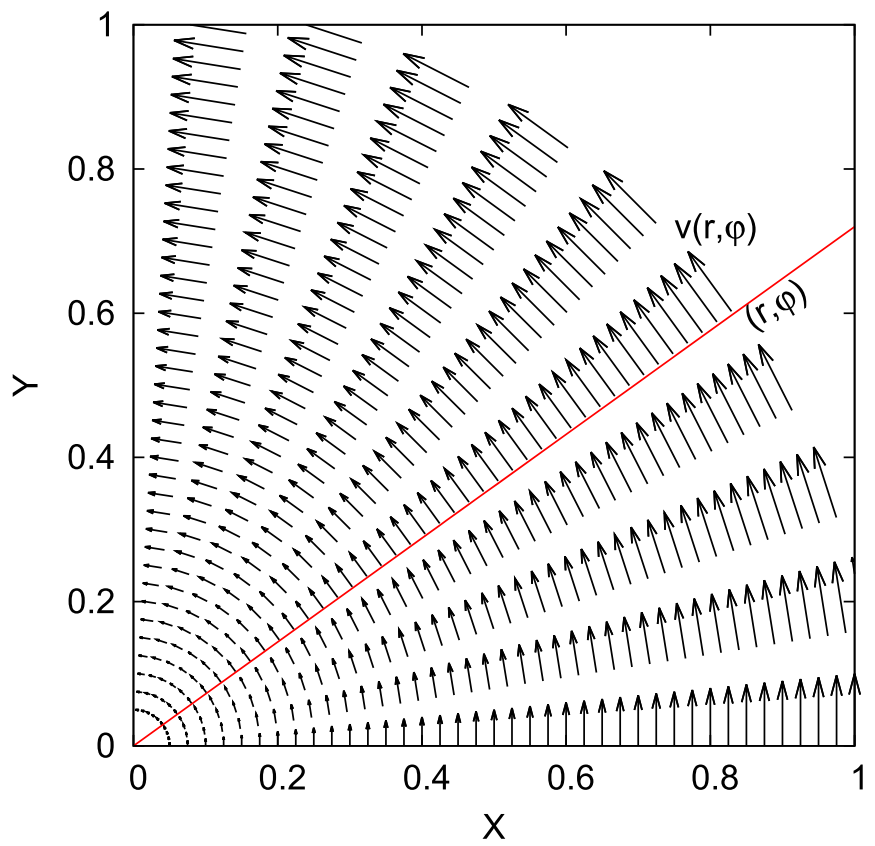

Figure 14. Sketch of the toy model used to estimate the characteristic turnover particle velocity. The figure shows the velocity field calculated with Equation (8) and $n=1$ as seen in the comoving frame of a particle located at the coordinate origin.

rotating single bubble is $\simeq 1.5 \times 10^{50} \mathrm{erg}$. At the moderate angular velocities considered in this work the estimated value of $v(h)$ is practically independent of $\Omega$.

\section{REFERENCES}

Blinnikov, S. I., Dunina-Barkovskaya, N. V., \& Nadyozhin, D. K. 1996, ApJS, 106, 171

Branch, D., \& Khokhlov, A. M. 1995, PhR, 256, 53

Bravo, E., \& García-Senz, D. 2006, ApJL, 642, L157

Bravo, E., \& García-Senz, D. 2008, A\&A, 478, 843

Bravo, E., \& García-Senz, D. 2009, ApJ, 695, 1244

Bravo, E., García-Senz, D., Cabezón, R. M., \& Domínguez, I. 2009, ApJ, 695,1257

Cabezón, R. M., García-Senz, D., \& Bravo, E. 2004, ApJS, 151, 345

Cabezón, R. M., García-Senz, D., \& Escartín, J. A. 2012, A\&A, 545, A112

Cabezón, R. M., García-Senz, D., \& Relaño, A. 2008, JCoPh, 227, 8523

Damköhler, G. 1940, Elektrochem., 46, 601

Diehl, R., Siegert, T., Hillebrandt, W., et al. 2015, A\&A, 574, A72

Domínguez, I., Piersanti, L., Bravo, E., Tornambé, A., \& Straniero, A. 2006, ApJ, 644, 21

Dursi, L. J., \& Timmes, F. X. 2006, ApJ, 641, 1071

Fink, M., Kromer, M., Seitenzahl, I., et al. 2014, MNRAS, 438, 1762

Gamezo, V. N., Khokhlov, A. M., \& Oran, E. S. 2005, ApJ, 623, 337

Gamezo, V. N., Wheeler, J. C., Khokhlov, A. M., \& Oran, E. S. 1999, ApJ, 512,827

García-Senz, D., \& Bravo, D. 2005, A\&A, 430, 585

García-Senz, D., Cabezón, R. M., Arcones, A., Relaño, A., \& Thielemann, F. K. 2013, MNRAS, 436, 3413

García-Senz, D., Cabezón, R. M., \& Escartín, J. A. 2012, A\&A, 538, A9

Harlow, F. H., \& Pracht, W. E. 1966, PhFl, 9, 1951

Hillebrandt, W., Kromer, M., Röpke, F. K., \& Ruiter, A. J. 2013, FrPhy, 8, 116

Hillebrandt, W., \& Niemeyer, J. C 2000, ARA\&A, 38, 191

Iben, I., Jr., \& Tutukov, A. V. 1984, ApJS, 54, 335

Isern, J., Jean, P., Bravo, E., et al. 2016, arXiv:1602.02918

Ivanova, L. N., Imshennik, V. S., \& Chechetkin, V. M. 1974, Ap\&SS, 31, 497

Iwamoto, K., Brachwitz, F., Nomoto, K., et al. 1999, ApJS, 125, 439

Jordan, G. C., IV, Fisher, R. T., Townsley, D. M., et al. 2008, ApJ, 681, 1448

Khokhlov, A. M. 1991a, A\&A, 245, 114

Khokhlov, A. M. 1991b, A\&A, 245, L25

Khokhlov, A. M. 1995, ApJ, 449, 695

Kuhlen, M., Woosley, S. E., \& Glatzmaier, G. A. 2006, ApJ, 640, 407 
Lorén-Aguilar, P., Isern, J., \& García-Berro, E. 2009, A\&A, 500, 1193

Ma, H., Woosley, S. E., Malone, C. M., Almgren, A., \& Bell, J. 2013, ApJ, 771,58

Malone, C. M., Nonaka, A., Woosley, S. E., et al. 2014, ApJ, 782, 11

Meakin, C. A., Seitenzahl, I., Townsley, D., et al. 2009, ApJ, 693, 1188

Niemeyer, J. C., \& Woosley, S. E. 1997, ApJ, 475, 740

Nomoto, K. 1982, ApJ, 253, 798

Nomoto, K., Thielemann, F.-K., \& Yokoi, K. 1984, ApJ, 286, 644

Nonaka, A., Aspden, A. J., Zingale, M., et al. 2012, ApJ, 745, 73

Pakmor, R., Kromer, M., Taubenberger, S., \& Springel, V. 2013, ApJL, 770, L8

Pfannes, J. M. M., Niemeyer, J. C., \& Schmidt, W. 2010a, A\&A, 509, A75

Pfannes, J. M. M., Niemeyer, J. C., Schmidt, W., \& Klingenberg, C. 2010b, A\&A, 509, A74

Piersanti, L., Gagliardi, S., Iben, I., Jr., \& Tornambé, A. 2003, ApJ, 598, 1229

Piro, A. L. 2008, ApJ, 679, 616

Plewa, T. 2007, ApJ, 657, 942

Plewa, T., Calder, A. C., \& Lamb, D. Q. 2004, ApJL, 612, L37

Reinecke, M., Hillebrandt, W., Niemeyer, J. C., Klein, R., \& Gröbl, A. 1999, A\&A, 347, 724

Riess, A. G., Filippenko, A. V., Challis, P., et al. 1998, AJ, 116, 1009
Röpke, F. K., Woosley, S. E., \& Hillebrandt, W. 2007, ApJ, 660, 1344

Rosswog, S. 2009, NewAR, 53, 78

Rosswog, S., Kasen, D., Guillochon, J., \& Ramirez-Ruiz, E. 2009, ApJL, 705, L128

Seitenzahl, I., Meakin, C. A., Lamb, D. Q., \& Truran, J. W. 2009a, ApJ, 700,642

Seitenzahl, I., Meakin, C. A., Townsley, D. M., Lamb, D. Q., \& Truran, J. W. 2009b, ApJ, 696, 515

Seitenzahl, I. R., Ciaraldi-Schoolmann, F., Röpke, F.-K., et al. 2013, MNRAS, 429, 1156

Springel, V. 2010, ARA\&A, 48, 391

Springel, V., \& Hernquist, L. 2002, MNRAS, 333, 649

Steinmetz, M., Müller, E., \& Hillebrandt, W. 1992, A\&A, 254, 177

Tenorio-Tagle, G., Cantó, J., \& Rózyczka, M. 1988, A\&A, 202, 256

Timmes, F. X., \& Woosley, S. E. 1992, ApJ, 396, 649

Townsley, D. M., Calder, A. C., Asida, S. M., et al. 2007, ApJ, 668, 1118

Wang, B., \& Han, Z. 2012, NewAR, 56, 122

Whelan, J., \& Iben, I., Jr. 1973, ApJ, 186, 1007

Yoon, S. C., \& Langer, N. 2004, A\&A, 419, 623

Yoon, S. C., \& Langer, N. 2005, A\&A, 435, 967

Zingale, M., Nonaka, A., Almgren, A. S., et al. 2011, ApJ, 740, 8 